\title{
Ages for exoplanet host stars
}

Jørgen Christensen-Dalsgaard and Víctor Silva Aguirre

\begin{abstract}
Age is an important characteristic of a planetary system, but also one that is difficult to determine. Assuming that the host star and the planets are formed at the same time, the challenge is to determine the stellar age. Asteroseismology provides precise age determination, but in many cases the required detailed pulsation observations are not available. Here we concentrate on other techniques, which may have broader applicability but also serious limitations. Further development of this area requires improvements in our understanding of the evolution of stars and their age-dependent characteristics, combined with observations that allow reliable calibration of the various techniques.
\end{abstract}

\section{Introduction}

The age is an obviously important part of the characterization of an exoplanetary system. It determines the time available for the system's evolution, including the processes that have resulted in its present configuration; an example is the analysis by Triaud (2011) of the age dependence of orbital inclination, measured with the Rossiter-McLaughlin effect. Also, the age of the system sets the scene for the evolution of life on possible habitable planets. Determination of such ages must in almost all cases be based on inference of the age of the central star of the system. The so far only known exception is the solar system, where precise, and probably accurate, ages can be obtained from analysis of radioactive isotopes, even detailing the ages of individual components of the original planetary disk (e.g., Connelly

Jørgen Christensen Dalsgaard

Stellar Astrophysics Centre, Department of Physics and Astronomy, Aarhus University, Ny Munkegade 120, 8000 Aarhus C, Denmark, e-mail: jcdephys . au.dk

Victor Silva Aguirre

Stellar Astrophysics Centre, Department of Physics and Astronomy, Aarhus University, Ny Munkegade 120, 8000 Aarhus C, Denmark, e-mail: victorephys .au.dk 
et al. 2012). In contrast, age determination for distant stars is always indirect, depending on the observation of some property that changes with time, such that the determination will in the end always depend on stellar modelling and the associated potential systematic uncertainties. For stars in and just after the phase of central hydrogen burning, the so-called main sequence, the change in stellar properties is based on the gradual change in the composition of the stellar core as hydrogen fuses into helium. However, there are secondary changes in stellar properties, particularly related to the evolution of rotation and magnetic activity, which also provide potential age diagnostics. Extensive reviews of stellar age determination were provided by Soderblom (2010) and Jeffries (2014), while Soderblom et al. (2014) focussed on the challenges of ages of young stars.

As discussed in the chapter Characterizing host stars using asteroseismology (see also below) asteroseismic analysis of observations of stellar oscillations provides a relatively precise measure of stellar age which is directly related to stellar internal properties and hence to the nuclear evolution of the star. Space-based photometry with CoRoT and, in particular, Kepler, has been an excellent source of oscillation observations, leading to asteroseismic characterization, including determination of ages, for a number of exoplanet-host stars (e.g., Silva Aguirre et al. 2015). However, such observations of main-sequence stars require a rapid cadence and good signal-to-noise ratio, to detect the faint oscillation signal; also, stars cooler than about spectral type K generally do not show oscillations. As a result, asteroseismic age determinations have been limited to the brightest and best-observed Kepler targets. Ages of fainter or cooler stars must be determined with other methods, possibly calibrated with asteroseismic data. These methods are the focus of the present chapter.

\section{Observational properties of stars}

Here we focus on 'classical' properties obtained from photometric and spectroscopic observations, and their relation to the intrinsic stellar properties. For a detailed overview of astrophysical observations, see, for example, Chromey (2016). From a global perspective stellar models are characterized by the mass $M$, the surface radius $R$ and the surface luminosity $L$. In addition, the temperature in the stellar atmosphere is represented by the effective temperature $T_{\text {eff }}$ defined, according to the relation for a black body, by

$$
L=4 \pi R^{2} \sigma T_{\text {eff }}^{4},
$$

where $\sigma$ is the Stefan-Boltzmann constant. An important quantity is also the surface gravity

$$
g=\frac{G M}{R^{2}},
$$

where $G$ is the gravitational constant; when characterizing observations $g$ is most often represented by $\log g$, with $\log$ being $\log$ arithm to base 10 . Finally, the com- 
position is typically specified by the abundances $X, Y$ and $Z$ by mass of hydrogen, helium and elements heavier than helium, often called 'metals'; these quantities satisfy the obvious relation $X+Y+Z=1$.

Photometric observations are typically characterized in terms of magnitudes $m$, related to the observed flux $f$ by $m=-2.5 \log f+c$, where $c$ is a constant which in practice is determined from suitable standard stars. Here the observed flux depends on the properties of the instrument, including the choice of filters, the absorption in the atmosphere, and the intrinsic distribution of energy with wavelength in the stellar spectrum. Different magnitude scales are defined by different choices of filters, collected in systems designed to provide a reasonable characterization of the energy distribution. An example is the Johnson system with magnitudes denoted $U, B$ and $V$ sensitive to the ultraviolet, blue and visual parts of the spectrum, respectively. Since the energy distribution roughly follows a black-body (Planck) curve, differences between magnitudes in different bands, or colour indices such as $B-V$, provide a measure of $T_{\text {eff }}$. Also, from a properly calibrated determination of the magnitude, together with a bolometric correction relating this to the total energy received at Earth from the star and a determination from parallax measurement of its distance, the stellar total luminosity $L$ can be determined. Stellar distance determinations will be greatly improved by the very accurate parallax observations from the Gaia mission (see e.g., Gaia Collaboration et al.2016).

Spectroscopic observations determine the absorption-line profiles which provide a measure of the surface composition of the star. In cooler stars, typical of exoplanet hosts, lines of helium are not visible. Hence the composition is characterized by the ratio $Z_{\mathrm{s}} / X_{\mathrm{s}}$ between the abundance of heavy elements and hydrogen. Also, observations are often measured relative to the Sun, motivating the definition of the metallicity as

$$
[\mathrm{M} / \mathrm{H}]=\log \left(\frac{Z_{\mathrm{s}} / X_{\mathrm{s}}}{Z_{\mathrm{s}, \odot} / X_{\mathrm{s}, \odot}}\right) .
$$

Since the iron abundance is typically well characterized by the observations it is common to replace $[\mathrm{M} / \mathrm{H}]$ by $[\mathrm{Fe} / \mathrm{H}]$. It should be noted, however, that the transition from the observed $[\mathrm{Fe} / \mathrm{H}]$ to $Z_{\mathrm{s}} / X_{\mathrm{s}}$ requires knowledge of $Z_{\mathrm{s}, \odot} / X_{\mathrm{s}, \odot}$, which has undergone recent revisions (e.g., Asplund et al. 2009) leading to serious problems with solar modelling (for a review, see Basu and Antia 2008). An additional uncertainty is the helium abundance; this is often obtained, although with no strong justification, by relating it to $Z$ through an assumed ratio $\Delta Y / Z$ between the increase in $Y$ and $Z$ since big-bang nucleosynthesis, obtained from Galactic chemical evolution. The relative strengths of the spectral lines also depend on the ionization and excitation states of the atoms in the stellar atmosphere and hence provide a measure of $T_{\text {eff }}$, while the line broadening depends on the density and hence gravity in the stellar atmosphere, such that the line widths can be used to determine $\log g$. The detailed analyses to determine $T_{\text {eff }}, \log g$ and composition utilize fits to computed stellar atmosphere models. In the following we generally neglect the complications of the photometric and spectroscopic analyses and assume that the stars can be characterized by $T_{\text {eff }}, L$ (or $\log g$ ), and their surface metallicity. 
We finally note that stellar radii can be obtained from interferometric observations (e.g., Hanbury Brown et al. 1974, Segransan et al. 2003, Boyajian et al. 2012, and references therein), combined with determination of the distance of the star. This will only be available for a fairly limited sample of stars but plays an important role in the calibration of other techniques to characterize stellar properties (see e.g., Huber et al.2012; White et al. 2013, Casagrande et al.2014).

\section{Ages from stellar evolution}

Stellar evolution can be followed through numerical modelling, based on equations describing hydrostatic equilibrium, energy production and energy transport in stellar interiors, and resulting in a description of the changes in the surface and internal properties of the star as it ages. A comprehensive description of stellar evolution is provided by Kippenhahn et al. (2012). The depletion of hydrogen in the core of main-sequence stars causes the core to contract, increasing the temperature in the stellar interior and consequently the surface luminosity, as well as the surface radius. When hydrogen is used up at the centre of the star nuclear fusion continues in a shell around the resulting helium core. In this phase the surface radius increases strongly, resulting in a decrease in the surface temperature; the star evolves towards the redgiant branch where the evolution continues at nearly constant surface temperature, as the star evolves towards strongly increasing luminosity with increasing radius (see Eq. 1). The evolution of stars is typically illustrated in a Hertzsprung-Russell (or HR) diagram, as shown in Fig. 1, an alternative, particularly when comparing with observations where the luminosity $L$ is unknown, is the so-called Kiel diagram, where luminosity is replaced by $\log g$.

The relation between stellar observable properties and age is clearer in a plot of the so-called isochrones (see Fig. 2), which shows the location of models of a given age, but varying mass. The lowest curve marks the zero-age main sequence (ZAMS), where stars have just started hydrogen fusion in the core. With increasing age, stars of lower and lower mass have reached the end of the central hydrogen fusion, leading to the isochrones bending away from the ZAMS. The later parts of the isochrones, corresponding to phases where evolution is relatively rapid, closely resemble the evolution tracks in Fig. 1

From Fig. 2 it is in principle possible to determine the age of a star, given observational determination of its effective temperature and luminosity. The practical problems are illustrated by the two simulated observations marked in the figure, for a star roughly corresponding to the Sun and for a 2 Gyr star of mass $\sim 1.5 \mathrm{M}_{\odot}$, assuming realistic uncertainties in $T_{\text {eff }}$ and $L$. For the lower-mass case it is evident that models of essentially any age are consistent with the observed $\left(T_{\text {eff }}, L\right)$. In the higher-mass case a clearer age determination would be possible, although here there is some non-uniqueness concerning the evolutionary stage of the star. To these uncertainties must be added the errors in the observed surface composition of the star, 


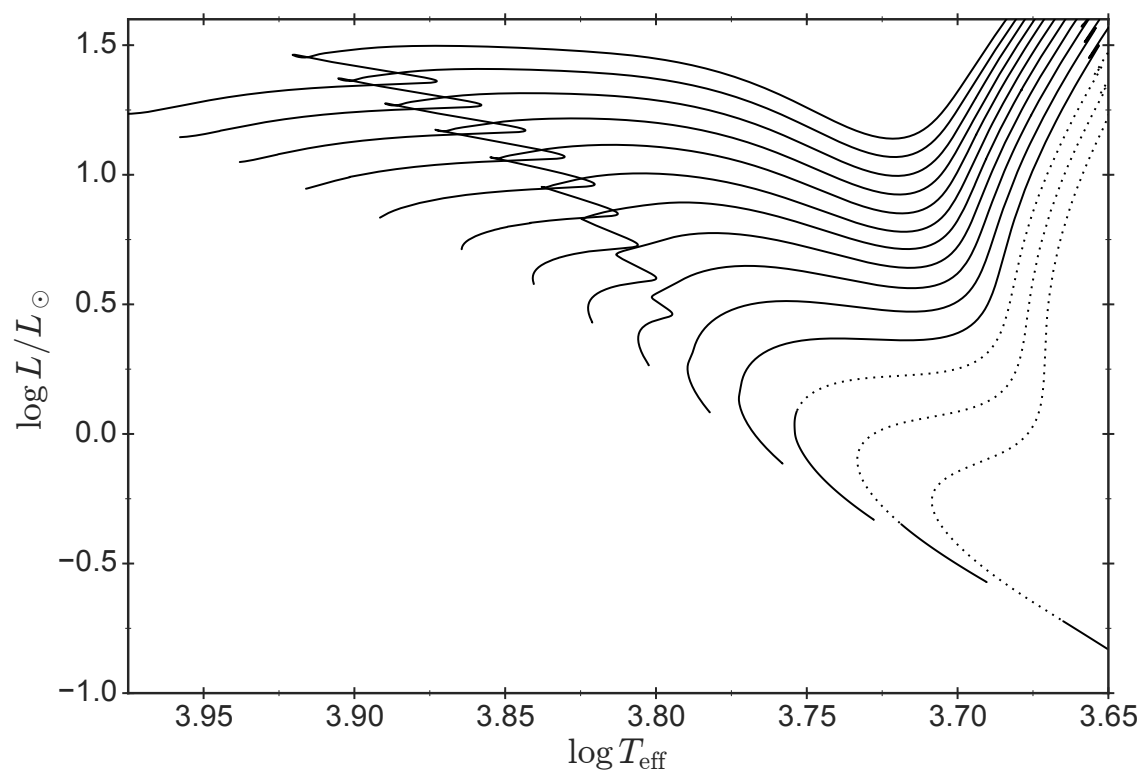

Fig. 1 Hertzsprung-Russell (or HR) diagram showing evolutionary tracks for masses between 0.7 and $2 \mathrm{M}_{\odot}$ in steps of $0.1 \mathrm{M}_{\odot}$. The portion of the tracks corresponding to ages greater than $14 \mathrm{Gyr}$ is shown as a dotted line.

which affects the location of the isochrones. It is evident that age determination, based on isochrones, is uncertain, particularly for stars at or below solar mass.

To sharpen the analysis, prior information may be included in age determination. An important aspect is the relative speed of evolution in the different evolutionary stages. In the example of the higher-mass star in Fig. 2 the choice of the $3 \mathrm{Gyr}$ isochrone would correspond to identifying the star to be in a phase of rapid evolution, where it would be less likely to be found. Also, one may take into account the fact that the fraction of stars increases rapidly with decreasing mass, reflecting the initial mass function. These aspects can be included through Bayesian analysis, where the assumed prior probability density is included in the estimate of final probability density function of, for example, the age (Pont and Eyer 2004; Jørgensen and Lindegren 2005, Serenelli et al. 2013).

An example portraying the difficulties in determining precise ages based on observed effective temperature and luminosity is shown in Fig. 3, depicting the position in the HR diagram of the sample of 33 Kepler exoplanet hosts analysed by Silva Aguirre et al. (2015). These targets were selected as they comprise the set of mainsequence stars harbouring planets with asteroseismic data of the highest quality that enables extraction of individual oscillation frequencies. As discussed in detail in the chapter Characterizing host stars using asteroseismology the inclusion of observed frequencies of solar-like oscillations in an asteroseismic analysis greatly constrains 


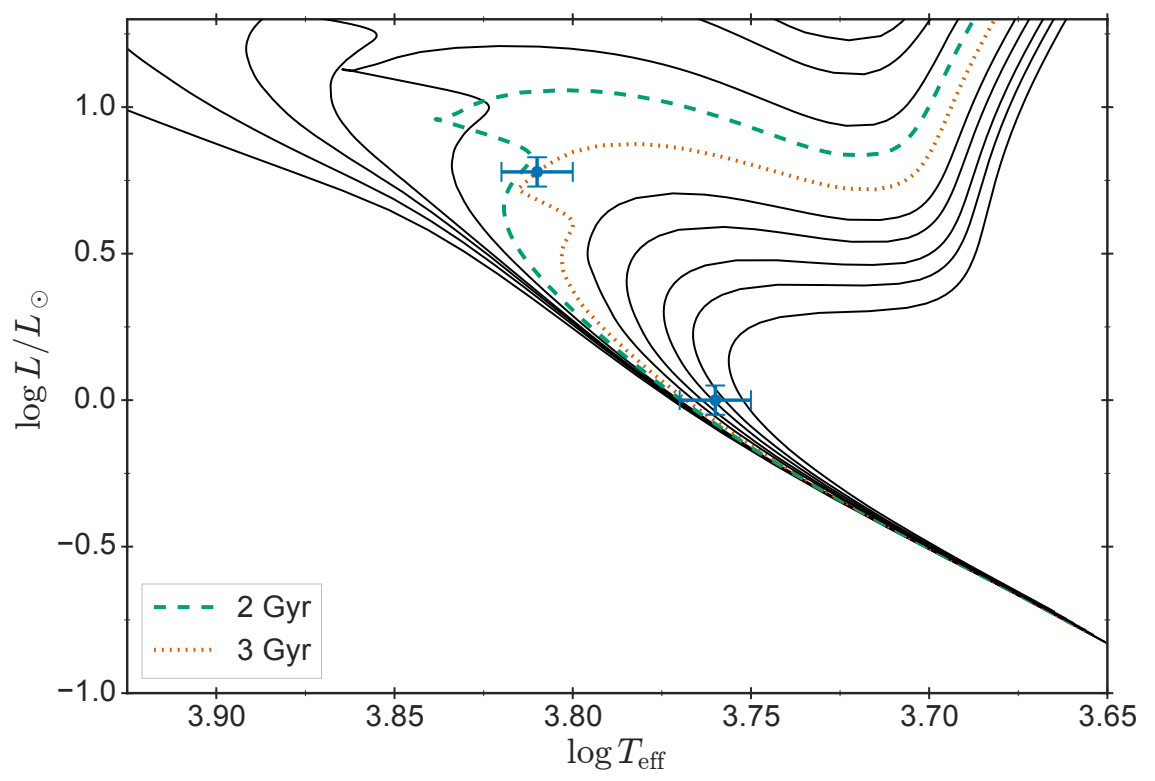

Fig. 2 Stellar isochrones at solar composition of ages (from left to right) 0, 0.5, 0.8, 1, 1.5, 2, 3, 4.6, 6, 8, 10, and $13 \mathrm{Gyr}$. The symbols represent the position of the Sun and that of a $1.48 \mathrm{M}_{\odot}$ star at $2 \mathrm{Gyr}$ of age. Plotted errors correspond to 0.01 in $\log T_{\text {eff }}$ and 0.1 in $\log L$. Dashed blue and dotted red lines depict the 2 and $3 \mathrm{Gyr}$ isochrones, respectively.

the inferred ages. Therefore, this set of stars serves as an excellent benchmark to compare the precision in age determination attainable from different methods.

The three panels of Fig. 4 display the resulting age uncertainties in the sample of 33 stars when different sets of observational data are fit to a grid of stellar models using a state-of-the-art Bayesian analysis scheme (BASTA, Silva Aguirre et al. 2015, 2017). In the absence of asteroseismic information, one can determine stellar properties using photospheric observables such as effective temperature, composition, and surface gravity (labelled spectroscopy). For the special case of stars with transiting exoplanets the analysis of the light curve can provide information about the mean density of the star, which adds very valuable constraints to the isochrone fit (Maxted et al. 2015a). If a strong constraint on the orbital eccentricity is available the stellar density can be extracted to a level of 5\% in the very best cases (e.g., Sandford and Kipping 2017), slightly improving the age determination as shown in the middle panel of Fig. 4 (labelled transits).

By far the most precise stellar ages for field stars are those resulting from the inclusion of asteroseismic information, where in addition to the effective temperature and composition the individual oscillation frequencies are fitted in BASTA (labelled asteroseismology in Fig. 4 . It is evident that for a large fraction of stars the age can be determined with a precision better than $20 \%$. Specific examples of interesting precise age determinations are the age of $10.4 \pm 1.4 \mathrm{Gyr}$ found for Kepler 


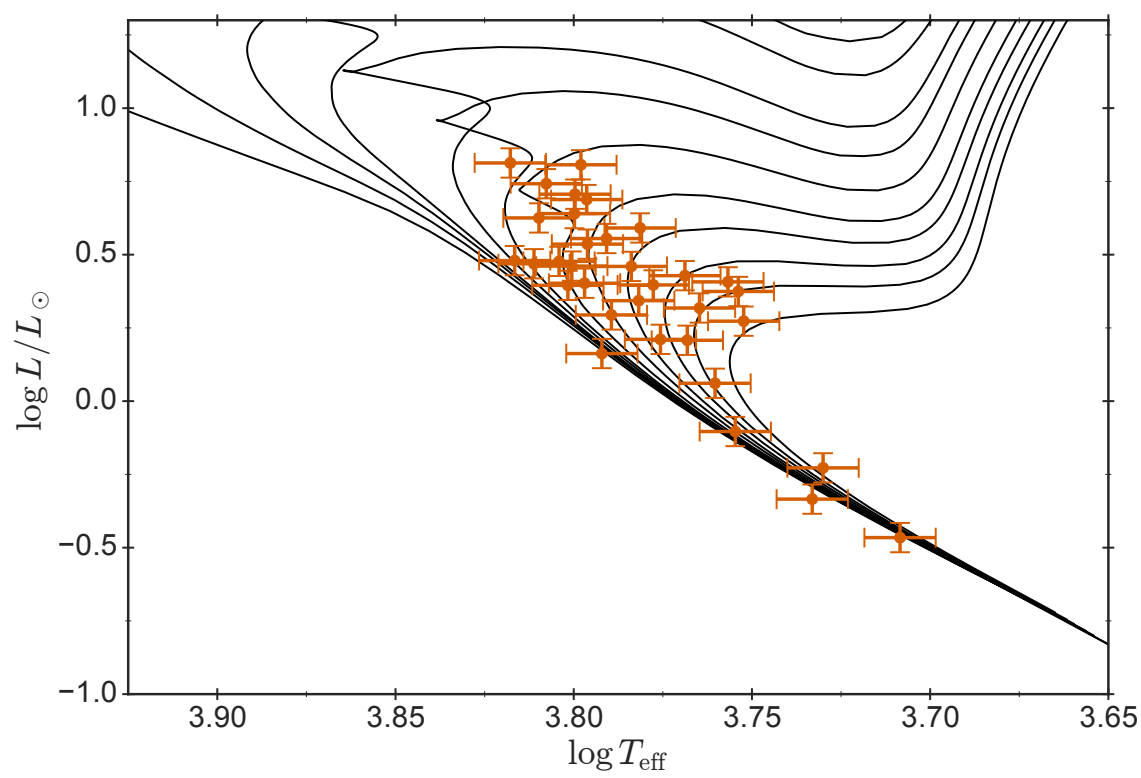

Fig. 3 Position in the HR diagram of the sample of 33 exoplanet host stars analysed by Silva Aguirre et al. (2015). Stellar isochrones are the same as those in Fig. 2 Uncertainties in $\log T_{\text {eff }}$ and $\log L$ correspond to 0.01 and 0.05 , respectively. See text for details.

10 (Fogtmann-Schulz et al. 2014) and the age of $11.2 \pm 1.0 \mathrm{Gyr}$ for the five-planet system around Kepler 444 (Campante et al. 2015). These results show that planetary systems were formed early in the history of the Milky Way Galaxy.

A special case of isochrone fits concerns stellar clusters. In such clusters the stars are normally assumed to have been formed at the same time and with the same initial composition, while covering a range of masses; hence they all have the same age, such that the distribution of stars in the $\left(T_{\text {eff }}, L\right)$ diagram should correspond to that of an isochrone. Fitting an isochrone, in particular the turn-off from the ZAMS, to the observations therefore provides a determination of the age of the cluster without the uncertainties associated with the fits of individual stars. Thanks to the K2 extension of the Kepler mission exoplanets have been found in the Hyades and Praesepe stellar clusters, both with an age of around $800 \mathrm{Myl}^{1}$ (Mann et al. 2016a, 2017, 2018), and in the very young Upper Scorpius OB Association (Mann et al. 2016b). This provides important information about the evolution of planetary systems with age. Also, well-determined ages of clusters play an important role in the calibration of other age-determination techniques. We return to this below.

These methods for age determination all rely on the modelling of stellar evolution and hence are sensitive to errors in the modelling. The basic theory, including the relevant physics of stellar matter, is probably fairly well established. However,

${ }^{1}$ According to Brandt and Huang 2015); more conventional ages are around $600 \mathrm{Myr}$ for both clusters. 


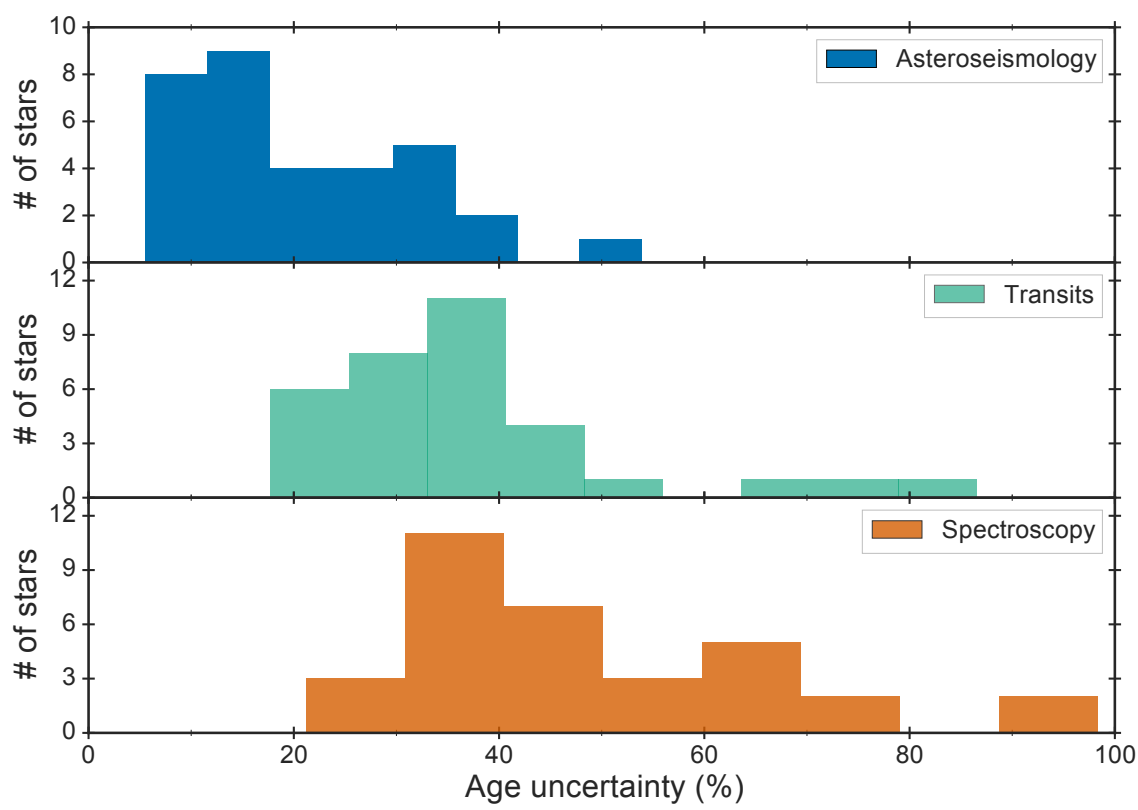

Fig. 4 Uncertainties in ages of exoplanet host stars determined with BASTA when fitting different sets of observational constraints. Top panel: individual oscillation frequencies, effective temperature, and metallicity. Middle panel: stellar density from the transit light curve, surface gravity, effective temperature, and metallicity. Bottom panel: surface gravity, effective temperature, and metallicity. See text for details.

there are serious uncertainties related to hydrodynamical instabilities and the resulting potential mixing processes. By changing the evolution of the core composition these would directly affect the relation between the age and the observable properties of the star. Main-sequence stars more massive than the Sun have a fully mixed convective core; a very uncertain aspect is extent of motion, the so-called convective overshoot, beyond the limit of convective instability, which may have important effects on stellar evolution. Constraints on overshooting have been obtained from asteroseismic analyses of main-sequence stars (Silva Aguirre et al. 2013, Deheuvels et al. 2016) as well as eclipsing binaries (Claret and Torres 2016), offering hope for a better understanding of this contribution to the age uncertainty. Mixing may also be induced by instabilities associated with the evolution of stellar rotation (see below).

\section{Ages from rotation and activity}

The rate of stellar rotation changes as stars evolve, making measurement of stellar rotation a potential age indicator. Stars are born as rapid rotators. In relatively cool 
stars with outer convection zones, supporting magnetic activity which is assumed to arise through some dynamo action and hence to be strongly linked to rotation, angular momentum is lost to a magnetic stellar wind which is coupled to the convection zone. This leads to a reduction of the surface rotation rate. Coupling to the radiative interior through processes that are not fully understood reduces the angular momentum of the interior, leading at least in the solar case to roughly uniform rotation (e.g., Thompson et al. 2003). As reviewed by Jeffries (2014) the evolution of rotation and hence magnetic activity with age provides potential for age determination.

Based on a small number of rotation determinations Skumanich (1972) inferred that the rotation rate decreases with the age $t$ of the star as $t^{-1 / 2}$, with a corresponding decrease in stellar activity. Durney (1972) independently carried out a simplified analysis of the angular-momentum loss, leading to the same time dependence. Thus a determination of stellar rotation rates, with proper calibration, can be used for age determination. This is the basis for gyrochronology (Barnes 2003, 2007).

As discussed above, stellar clusters provide samples of stars with the same (and in many cases well-estimated) age and hence are an excellent basis for calibrating techniques for age determination. Figure 5 shows rotation periods for two clusters of different age plotted against colour index; this is a measure of the effective temperature and hence, at the cluster age, a measure of stellar mass, with the colour index increasing with decreasing mass. It is evident that the bulk of the stars in each case lie near a well-defined curve, with rotation period increasing with increasing $B-V$ and hence decreasing mass. The interpretation is that the loss of angular momentum through the magnetic stellar wind leads to a well-defined relation between age and rotation period, but at a rate that depends on the mass. The relatively few rapidlyrotating stars may yet have to reach this relation, possibly because of very rapid initial rotation or a different configuration of their magnetic field (Brown|2014).

Two main observational techniques are available for the determination of stellar rotation periods. Rotation contributes to the line broadening through the Doppler effect due to the line-of-sight component of the rotational velocity $v_{\text {rot }}$; thus only $v_{\text {rot }} \sin i$ is determined, where $i$ is the inclination of the rotation axis relative to the line of sight. Stellar rotation can also in many cases be determined from periodic variations in the luminosity of spotted stars. Nevertheless, the extraction of rotation periods from both the width of spectral lines and from the light modulation induced by starspots becomes increasingly difficult with increasing age and hence increasing rotation period. The rotational broadening of the spectral lines becomes comparable with the natural line width and hence difficult to isolate, and the decreasing magnetic activity level decreases the magnitude of the starspot modulation of the stellar intensity.

The high precision of space photometry, in particular with Kepler, has allowed the determination of rotation periods for a wide variety of stars. In particular, Meibom et al. (2011a, 2015) extracted rotation periods for stars in the two clusters NGC 6811 and NGC 6819, of age 2.1 and $2.5 \mathrm{Gyr}$, respectively. Figure 6 illustrates the close relation found between colour and rotation period for NGC 6819. On this

2 Note that since, as shown in Fig. 5 the rotation periods also depend on the colour of the stars, a more proper term would be gyrochromochronology. 

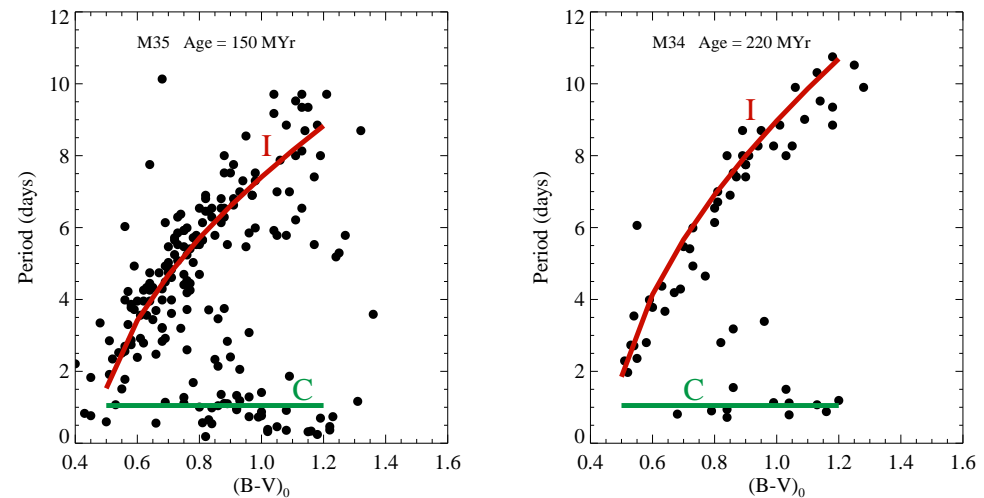

Fig. 5 Rotation periods as a function of (B-V) colour for stars belonging to two open clusters. The $C$ and $I$ sequences correspond to Convective and Interface magnetic fields as defined by Barnes (2003). From Brown (2014), using data from Meibom et al. (2009. 2011b) (c)AAS. Reproduced with permission.

basis Meibom et al. (2015) could extend the $t^{-1 / 2}$ dependence of rotation on age (Skumanich 1972) to a broader range, as illustrated schematically in Fig 7, where the Sun is used to anchor the relation at $4.57 \mathrm{Gyr}$. The relation was further extended by Barnes et al. (2016) to nearly solar age using K2 observations of the $4 \mathrm{Gyr}$ old cluster M67.

Few open clusters are older than M 67, and for these determinations of rotation periods have not been possible. However, the Kepler observations have provided rotation-period determinations and asteroseismic age inferences for a number of stars, many of which are older (van Saders et al. 2016). The results are illustrated in Fig. 8, comparing them with the fit provided by Meibom et al. (2015). Strikingly, there are very substantial deviations, with stars older than the Sun in most cases rotating considerably faster than predicted by the simple $t^{-1 / 2}$ variation, indicating a decrease in the efficiency of the angular-momentum loss. van Saders et al. (2016) speculate that this is related to the evolution with age of the so-called Rossby number Ro, i.e., the ratio between the rotation period and the convective turn-over timescale, and perhaps related to a change of the magnetic topology from being predominantly dipolar to a more complex structure. It appears that the departure from the simple age dependence sets in when Ro exceeds a critical value of around 2; interestingly, 


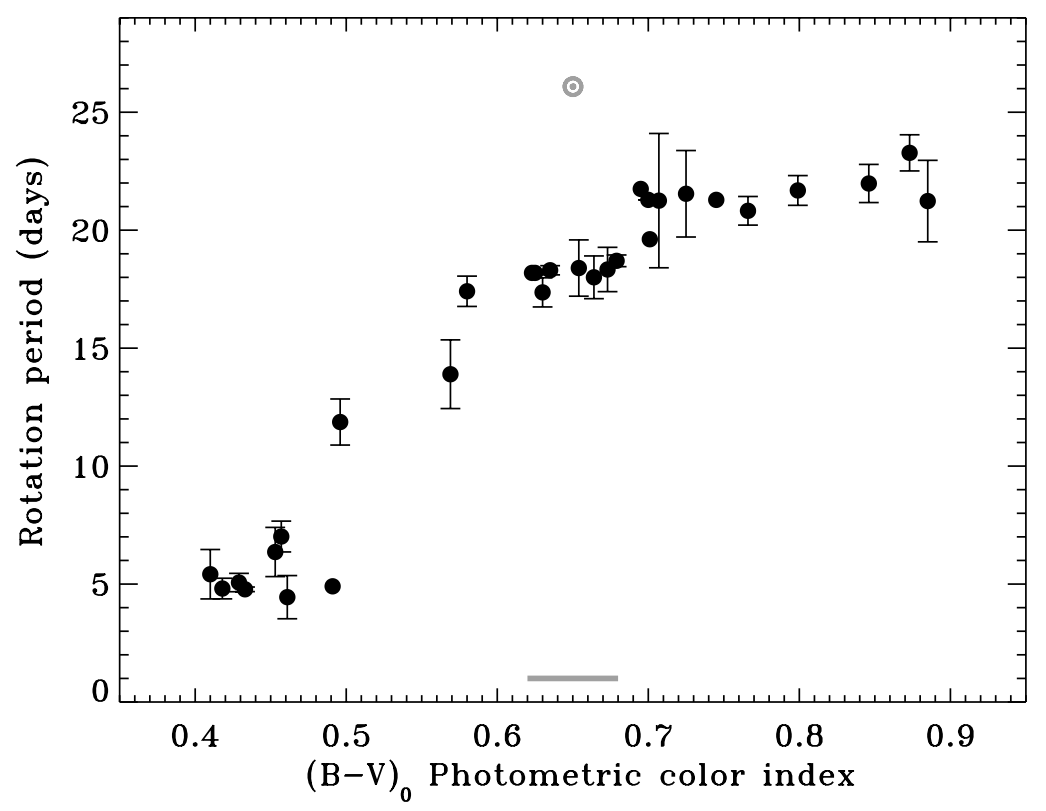

Fig. 6 Rotation periods as a function of (B-V) photometric index for a sample of stars in the open cluster NGC 6819 observed by Kepler. Stellar masses are given in the top of the figure while the position of the Sun in this diagram is shown by the solar symbol. From Meibom et al. (2015), (C) Nature. Reproduced with permission.

the Sun is close to this limit. These issues are extremely interesting in the context of the origin and evolution of stellar magnetic activity, but for age determination based on gyrochronology they are clearly a complication which restricts the reliable use to relatively young stars, at least until we have a better understanding of the transition to weaker angular-momentum loss and its relation to observable stellar properties.

Given the close relation between stellar rotation and magnetic activity, observations sensitive to activity can also be used for age diagnostics. A commonly used index is obtained from the chromospheric emission in the cores of the singly-ionized calcium $\mathrm{H}$ and $\mathrm{K}$ lines, which are located in the near-ultraviolet. As shown by Skumanich (1972) this emission decreases with age, like the rotation, as $t^{-1 / 2}$. Mamajek and Hillenbrand (2008) made a careful analysis of the relation between age and the calcium index, considering also combining this with the information provided by the rotation rate. They estimated that the achievable precision when using these techniques in $\log t$ is around $0.1-0.2$, in the age range $0.6-4.5 \mathrm{Gyr}$, with worse precision for younger and older stars. Indeed, one might have expected that the departures from the Skumanich relation for rotation found by van Saders et al. (2016) in older stars would also affect the relation between age and magnetic activity. Stellar activity, inferred from X-ray observations of stellar coronae, was compared with 


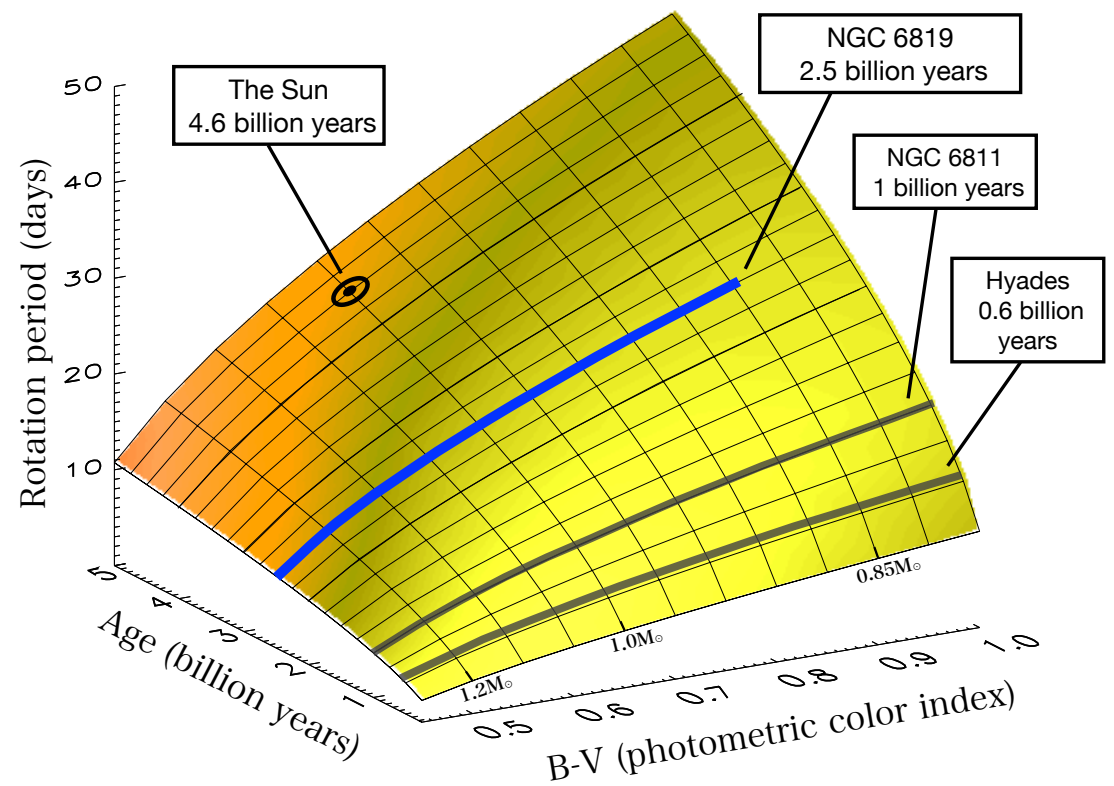

Fig. 7 Predicted surface depicting the relation between period, age, and colour index extrapolated to ages greater than that of NGC 6819 by anchoring it to the Sun. From Meibom et al. (2015), (c) Nature. Reproduced with permission.

asteroseismic ages by Booth et al. (2017). Interestingly, they found that the activity decreased more rapidly with age for stars older than the Sun than for younger stars, unlike the slower decrease of rotation with age inferred by van Saders et al. (2016). This suggests a more complex relation between rotation and activity than previously assumed, clearly further complicating the use of rotation and activity as age indicators.

\section{Ages from lithium abundance}

Lithium is created in big-bang nucleosynthesis and is destroyed by nuclear reactions at relatively modest temperature; in the case of the more abundant ${ }^{7} \mathrm{Li}$ isotope, which we consider in the following, destruction on a comparatively short timescale happens for temperatures above $2.5 \times 10^{6} \mathrm{~K}$. In the solar case the surface lithium abundance has been reduced by roughly a factor 150 compared with the assumed initial abundance. Thus the evolution with time of the surface lithium abundance provides a potential age diagnostics (see Jeffries 2014, for a review). Sestito and Randich (2005), for example, analysed lithium observations for a number of stellar 


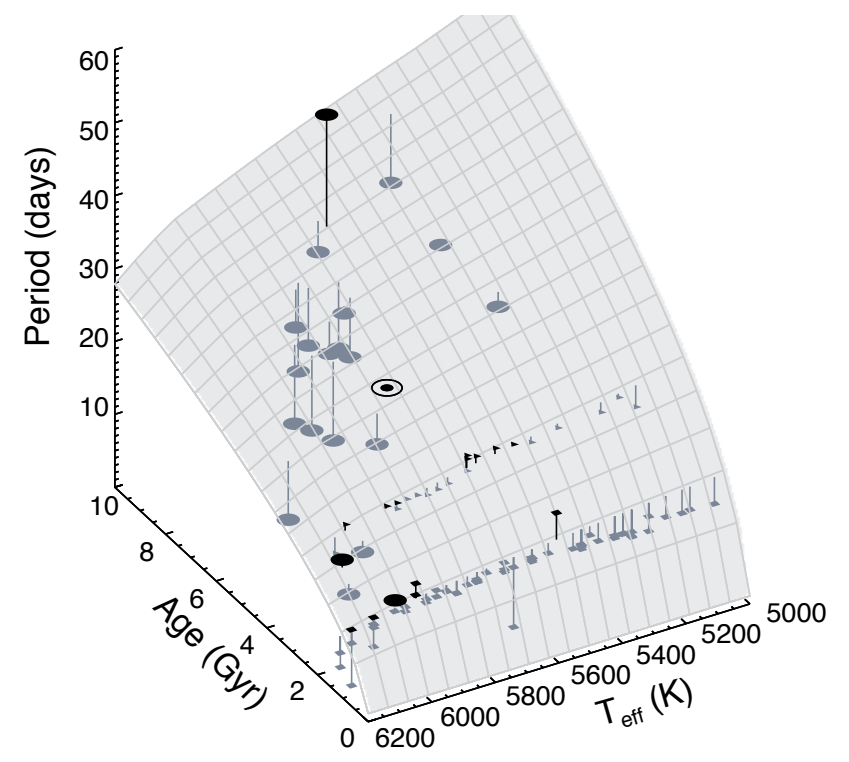

Fig. 8 Similar to Fig. 7 where asteroseismic ages of field stars with rotation periods measured from Kepler data are represented by filled circles. Squares and triangles depict the open clusters NGC 6811 and NGC 6819, respectively. From van Saders et al.(2016), (C) Nature. Reproduced with permission.

clusters to relate the abundance to age. However, the details of the processes that lead to lithium destruction are uncertain. In general, the outer convective envelope is not sufficiently deep to reach the temperatures required for lithium destruction. Thus, additional mixing below the convectively unstable region is required. This is likely related to the angular momentum transport that couples the decrease in the surface rotation rate, owing to the angular-momentum loss to a magnetic stellar wind, to the deeper interior of the star (e.g., Somers and Pinsonneault 2016). It appears that most of the lithium destruction takes place during the first few Gyr of the evolution, restricting the usefulness of lithium as an age diagnostics to relatively young stars.

\section{Conclusions}

In this chapter we have discussed various non-asteroseismic techniques for age determination in lower-main-sequence stars which are the most typical exoplanet hosts. None of these techniques can compete with asteroseismology, based on individually determined frequencies, in terms of precision and likely accuracy. On the other hand, with present and foreseeable observational data detailed asteroseismol- 
ogy will only be possible for a fraction of the stars for which exoplanets are found. Thus alternative techniques are essential. Stellar evolution and hence age is directly reflected by the location of the star in the HR diagram, allowing age determination through isochrone fitting, although with strong degeneracy, particularly for lowmass stars. Including the mean stellar density, as may be determined from transiting exoplanets, improves the resolution in the best cases when a strong density prior is available. Gyrochronology, based on the relation between rotation period, colour and age, is promising, at least for stars up to solar age. For exoplanets detected with the transit technique the determination of the rotation period from spot modulation will in many cases be a natural by-product of the observations. For older stars, with low activity, this signal becomes weaker and possibly hard to interpret, and in addition the relation between age and rotation period appears to undergo a transition which is so far not fully understood.

To test the validity of age inferences, comparisons of the results of different techniques are clearly very valuable. In the special case of isochrone fitting and gyrochronology for exoplanet hosts there seems to be some inconsistency, with gyrochronology in some cases indicating substantially lower ages, corresponding to faster rotation (e.g., Maxted et al.|2015b, and references therein). One proposed origin for this discrepancy, at least for stars with hot Jupiters, is that the rotation of the star has been spun up through tidal interaction with the close-in planet, which would clearly affect gyrochronology. Further studies are required to investigate this; it is obvious that asteroseismic ages for such systems would be of great value, to resolve the apparent conflict.

Very cool and low-mass main-sequence stars, the so-called $\mathrm{M}$ dwarfs, are of particular interest for exoplanet research: their small radii and masses make earth-size planets easier to detect, and their very low luminosities place the habitable zone close to the star, with orbital periods of only a couple of weeks, further increasing the chances of finding relevant planets. A remarkable example is the Trappist-1 system, with three potentially habitable planets (Gillon et al. 2017). Age determination for these systems is difficult. The nuclear evolution of such low-mass stars is extremely slow, such that the location of the star in the HR diagram provides little constraint on the age. Also, the predicted amplitudes of solar-like oscillations are very low, precluding asteroseismology. Burgasser and Mamajek (2017) carried out an extensive analysis of Trappist-1, including location in the HR diagram, mean density, rotation and lithium abundance, to infer an age of 7.6 $\pm 2.2 \mathrm{Gyr}$. Further work on ages of very low mass stars, e.g., based on stars in clusters with well-determined ages, would be very valuable.

Age determination based on nuclear evolution, including asteroseismology, depends directly on the reduction of the hydrogen abundance in the stellar core and hence is insensitive to the very interesting early phases of the life of a star, where nuclear burning is just starting. Here the variations in stellar rotation and activity may provide age information, although with very substantial uncertainties owing to a strong dependence on initial conditions and our incomplete understanding of the relevant processes. Further constraints may be available from lithium destruction, although again limited by uncertainty about the relevant mixing processes. For pre- 
main-sequence stars in the $\delta$ Scuti instability strip Zwintz et al. (2014) demonstrated a clear relation between the age and the oscillation properties, largely reflecting the changing radii of the stars as they contract towards the main sequence. This has the potential for further development towards a more sensitive technique for age determination in this case.

We emphasize again that the accuracy of any technique for age determination is limited by our incomplete understanding of the underlying processes, and, obviously, by the errors in the observations underlying the determination. It is fair to say that the nuclear evolution, and hence age determination depending on it, is quite well understood in broad outline, although substantial uncertainties remain, particularly regarding mixing processes in the stellar interior which directly affect the rate at which hydrogen is consumed. Here improvements will follow from more detailed asteroseismic investigations of stellar interiors, together with an improved understanding of the relevant hydrodynamical processes leading to mixing and implementation of this understanding in stellar-evolution calculations. It should be kept in mind that the solar case offers by far the best potential for detailed comparisons between models and observations; the fact that the most recent solar models, using updated surface abundances, fail this test is a serious concern with potential implications for other stars. This is also related to the effects of other uncertainties in the required determination of stellar composition, although these concerns are probably most relevant for the intrinsically precise asteroseismic determinations.

For other age determination techniques considered here the limitations in our knowledge about the underlying physical processes are substantially bigger. This is true for gyrochronology, where the rate of angular momentum loss from the outer layers and the coupling to the rotation in the interior are poorly understood. Similar uncertainties affect the use of stellar activity as an age indicator, given that even the magnetic activity of the Sun and its relation to the properties of rotation and convection are far from fully understood. More detailed modelling of these processes is obviously important, and further insight will be obtained from detailed observations of the surface magnetic configuration for stars of different age (e.g. Folsom et al. 2016, 2018). However, improvement of the reliability of rotation and activity as age indicators will also depend strongly on further calibration against other age determinations, in particular open-cluster fitting and asteroseismology. The understanding of the evolution of rotation also affects the use of lithium destruction as an age indicator, given the very strong sensitivity of lithium burning to the mixing processes in the stellar interior.

Needless to say, these developments require analysis of additional data. Much data remain to be studied in detail from the nominal Kepler mission, which for a considerable time will remain amongst the best available data. Also, new missions are on the way combining exoplanet research and stellar astrophysics. The TESS mission (Ricker et al.2016), to be launched in the spring of 2018, will provide data for a huge number of stars, although for shorter periods. Since these are typically brighter than the Kepler targets, there is a potential for more extensive follow-up observations, e.g., concerning the effects of rotation and chemical composition. Also, the PLATO mission (Rauer et al. 2014), with scheduled launch in 2026, will provide 
observations of a quality matching Kepler but for a much larger number of brighter stars, combining long time series and excellent opportunities for follow-up observations. This promises a breakthrough in the characterization of exoplanet hosts, including the determination of ages. To make the full use of this potential the analysis of the PLATO data must be organized to combine the exoplanet characterization with improvements in stellar modelling, concerning all the aspects discussed here, on the basis of the PLATO observations.

\section{Cross-References}

- Characterizing host stars using asteroseismology

- Rotation of Planet-Hosting Stars

Acknowledgements Funding for the Stellar Astrophysics Centre is provided by The Danish National Research Foundation (Grant DNRF106). V.S.A. acknowledges support from the Villum Foundation (Research grant 10118).

\section{References}

Asplund M, Grevesse N, Sauval AJ Scott P (2009) The Chemical Composition of the Sun. ARA\&A47:481-522

Barnes SA (2003) On the Rotational Evolution of Solar- and Late-Type Stars, Its Magnetic Origins, and the Possibility of Stellar Gyrochronology. ApJ586:464-479

Barnes SA (2007) Ages for Illustrative Field Stars Using Gyrochronology: Viability, Limitations, and Errors. ApJ669:1167-1189

Barnes SA, Weingrill J, Fritzewski D, Strassmeier KG Platais I (2016) Rotation Periods for Cool Stars in the 4 Gyr old Open Cluster M67, The Solar-Stellar Connection, and the Applicability of Gyrochronology to at least Solar Age. ApJ823:16

Basu S Antia HM (2008) Helioseismology and solar abundances. Phys Rep 457:217-283

Booth RS, Poppenhaeger K, Watson CA, Silva Aguirre V Wolk SJ (2017) An improved age-activity relationship for cool stars older than a gigayear. MNRAS471:1012-1025

Boyajian TS, McAlister HA, van Belle G et al. (2012) Stellar diameters and temperatures. I. Mainsequence A, F, and G stars. ApJ 746(1):101

Brandt TD Huang CX (2015) The Age and Age Spread of the Praesepe and Hyades Clusters: a Consistent, 200 Myr Picture from Rotating Stellar Models. ApJ807:24

Brown TM (2014) The Metastable Dynamo Model of Stellar Rotational Evolution. ApJ789:101

Burgasser AJ Mamajek EE (2017) On the Age of the TRAPPIST-1 System. ApJ845:110

Campante TL, Barclay T, Swift JJ et al. (2015) An Ancient Extrasolar System with Five Sub-Earthsize Planets. ApJ799:170

Casagrande L, Portinari L, Glass IS et al. (2014) Towards stellar effective temperatures and diameters at 1 per cent accuracy for future surveys. Monthly Notices of the Royal Astronomical Society 439(2):2060-2073

Chromey FR (2016) To Measure the Sky

Claret A Torres G (2016) The dependence of convective core overshooting on stellar mass. A\&A592:A15 
Connelly JN, Bizzarro M, Krot AN et al. (2012) The Absolute Chronology and Thermal Processing of Solids in the Solar Protoplanetary Disk. Science 338:651

Deheuvels S, Brandão I, Silva Aguirre V et al. (2016) Measuring the extent of convective cores in low-mass stars using Kepler data: toward a calibration of core overshooting. A\&A589:A93

Durney B (1972) Evidence for Changes in the Angular Velocity of the Surface Regions of the Sun and Stars - Comments. NASA Special Publication 308:282

Fogtmann-Schulz A, Hinrup B, Van Eylen V et al. (2014) Accurate Parameters of the Oldest Known Rocky-exoplanet Hosting System: Kepler-10 Revisited. ApJ781:67

Folsom CP, Petit P, Bouvier J et al. (2016) The evolution of surface magnetic fields in young solartype stars - I. The first 250 Myr. MNRAS457:580-607

Folsom CP, Bouvier J, Petit P et al. (2018) The evolution of surface magnetic fields in young solar-type stars II: the early main sequence (250-650 Myr). MNRAS474:4956-4987

Gaia Collaboration, Brown AGA, Vallenari A et al. (2016) Gaia Data Release 1. Astronomy and Astrophysics 595:A2

Gillon M, Triaud AHMJ, Demory BO et al. (2017) Seven temperate terrestrial planets around the nearby ultracool dwarf star TRAPPIST-1. Nature542:456-460

Hanbury Brown R, Davis J Allen LR (1974) The Angular Diameters of 32 Stars. MNRAS167:121136

Huber D, Ireland MJ, Bedding TR et al. (2012) Fundamental properties of stars using asteroseismology from Kepler and CoRoT and interferometry from the CHARA Array. ApJ 760(1):32

Jeffries RD (2014) Using rotation, magnetic activity and lithium to estimate the ages of low mass stars. In: EAS Publications Series, EAS Publications Series, vol 65, pp 289-325, DOI 10.1051/ eas/1465008

Jørgensen BR Lindegren L (2005) Determination of stellar ages from isochrones: Bayesian estimation versus isochrone fitting. A\&A436:127-143

Kippenhahn R, Weigert A Weiss A (2012) Stellar Structure and Evolution. DOI 10.1007/ 978-3-642-30304-3

Mamajek EE Hillenbrand LA (2008) Improved Age Estimation for Solar-Type Dwarfs Using Activity-Rotation Diagnostics. ApJ687:1264-1293

Mann AW, Gaidos E, Mace GN et al. (2016a) Zodiacal Exoplanets in Time (ZEIT). I. A Neptunesized Planet Orbiting an M4.5 Dwarf in the Hyades Star Cluster. ApJ818:46

Mann AW, Newton ER, Rizzuto AC et al. (2016b) Zodiacal Exoplanets in Time (ZEIT). III. A Short-period Planet Orbiting a Pre-main-sequence Star in the Upper Scorpius OB Association. AJ152:61

Mann AW, Gaidos E, Vanderburg A et al. (2017) Zodiacal Exoplanets in Time (ZEIT). IV. Seven Transiting Planets in the Praesepe Cluster. AJ153:64

Mann AW, Vanderburg A, Rizzuto AC et al. (2018) Zodiacal Exoplanets in Time (ZEIT). VI. A Three-planet System in the Hyades Cluster Including an Earth-sized Planet. AJ155:4

Maxted PFL, Serenelli AM Southworth J (2015a) Bayesian mass and age estimates for transiting exoplanet host stars. A\&A575:A36

Maxted PFL, Serenelli AM Southworth J (2015b) Comparison of gyrochronological and isochronal age estimates for transiting exoplanet host stars. A\&A577:A90

Meibom S, Mathieu RD Stassun KG (2009) Stellar Rotation in M35: Mass-Period Relations, SpinDown Rates, and Gyrochronology. ApJ695:679-694

Meibom S, Barnes SA, Latham DW et al. (2011a) The Kepler Cluster Study: Stellar Rotation in NGC 6811. ApJ733:L9

Meibom S, Mathieu RD, Stassun KG, Liebesny P Saar SH (2011b) The Color-period Diagram and Stellar Rotational Evolution-New Rotation Period Measurements in the Open Cluster M34. ApJ733:115

Meibom S, Barnes SA, Platais I et al. (2015) A spin-down clock for cool stars from observations of a 2.5-billion-year-old cluster. Nature517:589-591

Pont F Eyer L (2004) Isochrone ages for field dwarfs: method and application to the age-metallicity relation. MNRAS351:487-504 
Rauer H, Catala C, Aerts C et al. (2014) The PLATO 2.0 mission. Experimental Astronomy 38:249-330

Ricker GR, Vanderspek R, Winn J et al. (2016) The Transiting Exoplanet Survey Satellite. In: Space Telescopes and Instrumentation 2016: Optical, Infrared, and Millimeter Wave, Proc SPIE, vol 9904, p 99042B, DOI 10.1117/12.2232071

Sandford E Kipping D (2017) Know the Planet, Know the Star: Precise Stellar Densities from Kepler Transit Light Curves. AJ154:228

Segransan D, Kervella P, Forveille T Queloz D (2003) First radius measurements of very low mass stars with the VLTI. Astronomy and Astrophysics 397(3):L5-L8

Serenelli AM, Bergemann M, Ruchti G Casagrande L (2013) Bayesian analysis of ages, masses and distances to cool stars with non-LTE spectroscopic parameters. MNRAS429:3645-3657

Sestito P Randich S (2005) Time scales of Li evolution: a homogeneous analysis of open clusters from ZAMS to late-MS. A\&A442:615-627

Silva Aguirre V, Basu S, Brandão IM et al. (2013) Stellar Ages and Convective Cores in Field Main-sequence Stars: First Asteroseismic Application to Two Kepler Targets. ApJ769:141

Silva Aguirre V, Davies GR, Basu S et al. (2015) Ages and fundamental properties of Kepler exoplanet host stars from asteroseismology. MNRAS452:2127-2148

Silva Aguirre V, Lund MN, Antia HM et al. (2017) Standing on the Shoulders of Dwarfs: the Kepler Asteroseismic LEGACY Sample. II.Radii, Masses, and Ages. ApJ835:173

Skumanich A (1972) Time Scales for CA II Emission Decay, Rotational Braking, and Lithium Depletion. ApJ171:565

Soderblom DR (2010) The Ages of Stars. ARA\&A48:581-629

Soderblom DR, Hillenbrand LA, Jeffries RD, Mamajek EE Naylor T (2014) Ages of Young Stars. Protostars and Planets VI pp 219-241

Somers G Pinsonneault MH (2016) Lithium Depletion is a Strong Test of Core-envelope Recoupling. ApJ829:32

Thompson MJ, Christensen-Dalsgaard J, Miesch MS Toomre J (2003) The Internal Rotation of the Sun. ARA\&A41:599-643

Triaud AHMJ (2011) The time dependence of hot Jupiters' orbital inclinations. A\&A534:L6

van Saders JL, Ceillier T, Metcalfe TS et al. (2016) Weakened magnetic braking as the origin of anomalously rapid rotation in old field stars. Nature529:181-184

White TR, Huber D, Maestro V et al. (2013) Interferometric radii of bright Kepler stars with the CHARA Array: Cygni and 16 Cygni A and B. MNRAS 433(2):1262-1270

Zwintz K, Fossati L, Ryabchikova T et al. (2014) Echography of young stars reveals their evolution. Science 345:550-553 